\title{
Mature follow-up of Low-dose rate interstitial brachytherapy following ipsilateral breast tumor recurrence in patients initially treated with breast conservation therapy
}

\author{
Mark Trombetta ${ }^{1,3}$, Thomas J ulian ${ }^{2,3}$, Yongbok Kim ${ }^{1,3}$, E. Day Werts ${ }^{1,3}$, David Parda ${ }^{1,3}$ \\ 1. Department of Radiation Oncology, Allegheny General Hospital, 320 East North Avenue, Pittsburgh, PA 15212. \\ 2. Department of Surgery, Allegheny General Hospital, Pittsburgh, PA 15212. 3. Drexel University College of Medicine, \\ Allegheny Campus, Pittsburgh, PA 15212
}

Correspondence: Mark Trombetta. Address: Department of Radiation Oncology, Allegheny General Hospital 320 East North Ave, Pittsburgh, PA 15212. Telephone: 1-412-359-3400. Fax: 1-412-359-3981. Email: mtrombet@wpahs.org

Received: September 10, 2011 Accepted: November 10, 2011 Published: February 1, 2012

DOI : 10.5430/jst.v2n1p8 URL: http://dx.doi.org/10.5430/jst.v2n1p8

\section{Abstract}

Objective: To evaluate our mature follow-up data in women who have developed IBTR following conservation surgery and post-operative external radiotherapy retreated by lumpectomy followed by interstitial brachytherapy in lieu of salvage mastectomy.

Methods: Between 1/1998 and 10/2006, twenty-two patients with TIS or T1 IBTR were offered interstitial low-dose rate brachytherapy following tumor re-excision as an alternative to salvage mastectomy. All patients had failed lumpectomy followed by standard postoperative external beam radiotherapy (range 5000-6480cGy). The recurrent tumors were excised with final margins of resection free of residual disease. Tumor bed implantation was then carried out with an interstitial technique utilizing ${ }^{192}$ Iridium with the target volume consisting of the tumor bed plus a minimum 1.0 centimeter margin (minimum standard dose 4500cGy).

Results: With a mean follow up of 67.5 months (range 17-115 months) Twenty-one of 22 patients maintained local control at the time of last follow-up or at the time of their death. The single patient who developed a second local recurrence was treated successfully with mastectomy. Three patients succumbed to systemic disease. Two patients developed localized skin breakdown. One patient developed a contralateral breast cancer. Long-term cosmetic results as defined by the Harvard cosmesis scale and the Allegheny General modification were acceptable.

Conclusions: These long-term data suggest that lumpectomy followed by brachytherapy is feasible and may be an acceptable alternative to salvage mastectomy in patients who locally fail conservation breast therapy. The upcoming RTOG retreatment trial (RTOG 1014) should help define the role of repeat conservation therapy in IBTR. 


\section{Key words}

Ipsilateral breast tumor recurrence, Conservation surgery, Conservation therapy, Mastectomy

\section{Introduction}

The two landmark studies establishing lumpectomy and post-operative radiotherapy as the standard of care for breast conservation have recently been updated with more than 20 years of follow-up ${ }^{[1,2]}$ confirming limited surgery and post-operative radiotherapy as equivalent to mastectomy in the management of early stage breast cancer. Both of these studies demonstrated long term in-breast recurrence rates of up to $14 \%^{[2]}$. The accepted standard of care for ipsilateral beast tumor recurrence (IBTR) has been salvage mastectomy, which has demonstrated excellent salvage local control rates of greater than $90 \%{ }^{[3,4]}$, but leaves women with a poor cosmetic and suboptimal functional result ${ }^{[5]}$.

The rationale for less than whole breast irradiation for IBTR emanates from the growing body of accelerated partial breast irradiation (APBI) data as an emerging standard of care and the understanding of long-term complication rates that are directly related to volume of tissue treated. Six clinical trials with a minimum 5 year mean follow-up including at least 40

patients ${ }^{[6-10]}$ have demonstrated efficacy of partial breast radiotherapy and suggest equivalence to mastectomy. Two others reported APBI with more than 10 years follow-up which demonstrate local control rates of $91-95 \%{ }^{[11,12]}$.

We and other groups have reported our initial experience with retreatment low-dose rate interstitial brachytherapy (LDR) following IBTR ${ }^{[13-15]}$ which demonstrated acceptable local control rates and cosmesis in properly selected patients. Chadha et al. have published dose escalation data demonstrating mastectomy-free local control in 93\% (14/15) of patients at a median follow-up of 36 months ${ }^{[14]}$. The initial 6 patients received a LDR dose of 3000 cGy prior to a dose escalation to 4500 cGy once the initial cohort demonstrated acceptable toxicity. The lone recurrence patient was treated with only 3000 cGy in the early arm of the study. These patients exhibited similar local control to that noted in the dose-escalation study of Hannoun-Levi et al. ${ }^{[15]}$, which used the same dose limits. Currently, the Radiation Therapy Oncology Group (RTOG) has opened protocol 1014 to study partial breast re-treatment radiotherapy ${ }^{[16]}$. This protocol is limited to only three-dimensional conformal hyper fractionated radiotherapy (3D-CRT) using 4500 cGy in 150 cGy fractions twice daily which has never been studied. With the data below, we present our long-term results of patients retreated with LDR brachytherapy following IBTR.

\section{Materials and methods}

Between 1/1998 and 10/2006, twenty-two patients with TIS or T1 IBTR underwent an in-house, Institutional Review Board approved, low dose rate (LDR) temporary interstitial implant procedure following re-excision as an alternative to salvage mastectomy. All patients had failed lumpectomy and postoperative whole breast external beam radiotherapy (range 5000-6040cGy). The median age at original diagnosis for all patients was 62 years (range 42 - 89 years). The mean time to initial failure was 9.58 years (range 0.4 - 46.0 years). Three patients were treated with systemic chemotherapy at initial diagnosis, while 17 received one or more hormone modulators (tamoxifen, anastrozole or both) during some part of their therapy. As a condition of entry into this study, all patients were required to be without evidence of distant metastatic disease or regional nodal disease as documented by physical examination, chest radiography or computed axial tomography, nuclear medicine bone scanning and comprehensive serum metabolic assay. The single patient who was known to have metastatic disease prior to implant had been treated with high dose chemotherapy and bone marrow transplantation as was a standard of care at the time. She had been clinically documented as disease free for two years prior to IBTR and had no other known disease. Since calendar year 2004, preoperative magnetic resonance imaging has been 
performed in all patients at our institution prior to repeat lumpectomy (6 patients). No patient with suspected multi-centric disease or contralateral breast lesions documented by mammography, sonography or magnetic resonance imaging was included in this study.

All patients in this study were informed that salvage mastectomy was the current standard of care and opted for a less aggressive management approach. If the implant was performed at the time of repeat lumpectomy (earlier patients), the recurrent tumor was excised following needle localization and, the intraoperative specimen margins were required to be negative by frozen section. Final negative margins of resection (defined by the NSABP standard as no tumor at the specimen edge) were also required for patient accession into this study. Sources were not loaded until final margin negativity was confirmed. At initial diagnosis, seven patients presented with ductal carcinoma in situ (DCIS), 10 with invasive ductal carcinoma, 2 with combination invasive carcinoma and DCIS, and 3 with infiltrating lobular carcinoma. Six patients recurred as DCIS, 10 as invasive ductal carcinoma, 4 as combination invasive carcinoma and DCIS, and 2 as infiltrating lobular carcinoma (one of these with a DCIS component). Surgical marking clips were used to identify the tumor bed radio graphically when possible.

\section{Treatment technique and planning}

The techniques for implantation of these patients have been previously described in detail elsewhere ${ }^{[13]}$. However, in general, freehand tumor bed implantation was performed using afterloading catheters with the clinical target volume (CTV) consisting of the tumor bed/seroma cavity plus a minimum $1.0 \mathrm{~cm}$ margin (Figure 1). The original technique was modified by limiting the seed placement in the catheters to a minimum $1.0 \mathrm{~cm}$ skin distance to reduce the integral skin dose after grade 3 (CTCAE v 4.0) ${ }^{[17]}$ skin reactions were noted in two patients. In 20 of 22 patients, implant volumes were confirmed with computerized tomographic scanning and three-dimensional reconstruction to analyze the volumetric parameters. The dosimetry of the first two cases was calculated utilizing orthogonal radiography. ${ }^{192}$ Iridium (mean activity $0.62 \mathrm{mg} \mathrm{Ra}$ eq) was used in an afterloading inpatient procedure to achieve a target total dose of 4500-5000cGy, (range 4500-5530cGy) (Figure 2). The mean V100 (volume of the implant receiving 100 percent of the prescribed dose) was 105 cc. (range 36-260 cc.). The mean V150 (volume of the implant receiving 150 percent of prescribed dose) was 30 cc. (range 1-105 сc.). The V200 (volume of the implant receiving 200 percent of prescribed dose) mean was 8cc. The average length of hospital stay was 4.5 days. Cosmetic result was assessed by the same attending physician using both the Harvard/NSABP criteria ${ }^{[18]}$ (Table I) and the Allegheny General Modification (Table II) ${ }^{[19]}$.

Table 1. Harvard/NSABP breast cosmesis grading scale

Excellent When compared to the untreated breast, there is minimal or no difference in the size or shape of the treated breast. The way the breasts feels (its texture) is the same or slightly different. There may be thickening, scar tissue, or fluid accumulation within the breast, but not enough to change the appearance.

Good There is a slight difference in the size or shape of the treated breast as compared to the opposite breast or the original appearance of the treated breast. There may be some mild reddening or darkening of the breast. The thickening or scar tissue within the breast causes only a mild change in the shape or size.

Fair Obvious difference in the size and shape of the treated breast. This change involves one quarter or less of the breast. There can be moderate thickening or scar tissue of the skin and the breast, and there may be obvious color changes.

Poor Marked change in the appearance of the treated breast involving more than one quarter of the breast tissue. The skin changes may be obvious and detract from the appearance of the breast. Severe scarring and thickening of the breast, which clearly alters the appearance of the breast, may be found. 
Table 2. The Allegheny general modification of the Harvard/RTOG/NSABP criteria

A-0 (X) $\begin{aligned} & \text { Where } \mathrm{X}=\text { the breast cosmesis grade according to the Harvard/RTOG/NSABP criteria prior to repeat surgical and } \\ & \text { radiotherapeutic intervention. }\end{aligned}$
A-1
When compared to the A-0 breast, there is minimal or no change in the size or shape of the retreated breast. The breast
texture is the same or slightly different. There may be thickening, scar tissue, or fluid accumulation within the breast, but
not enough to change the appearance.
When compared to the A-0 breast, there is a slight difference in the size or shape of the treated breast as compared to the
opposite breast or the original appearance of the retreated breast. There may be some mild reddening or darkening of the
breast. The thickening or scar tissue within the breast causes only a mild change in the shape or size.
When compared to the A-0 breast, there is an obvious difference in the size and shape of the treated breast. This change
involves one quarter or less of the breast. There can be moderate thickening or scar tissue of the skin and the breast, and
there may be obvious color changes.
When compared to the A-0 breast, there is marked change in the appearance of the treated breast involving more than
one quarter of the breast tissue. The skin changes may be obvious and detract from the appearance of the breast. Severe
scarring and thickening of the breast, which clearly alters the appearance of the breast, may be found.

The initial scoring should consist of the A0 (X) score and all subsequent scores should be graded $\mathrm{A} 0(\mathrm{X})$ followed by the post therapeutic A score [i.e., A (1-1) for excellent cosmesis pre and post retreatment]. Initial scoring for this same example would be graded as A (1-X) to denote the unknown future scoring.

Table 3. Improvement in reporting retreatment cosmetic outcomes using the Allegheny general modification of the Harvard criteria

\begin{tabular}{llll}
\hline $\mathbf{n}$ & Harvard & $\mathbf{n}$ & Allegheny general modification \\
\hline 13 & 1 & 13 & A $(1-1)$ \\
6 & 2 & 2 & A $(1-2)$ \\
3 & 3 & 4 & A $(2-2)$ \\
& & 3 & A $(2-3)$ \\
\hline
\end{tabular}

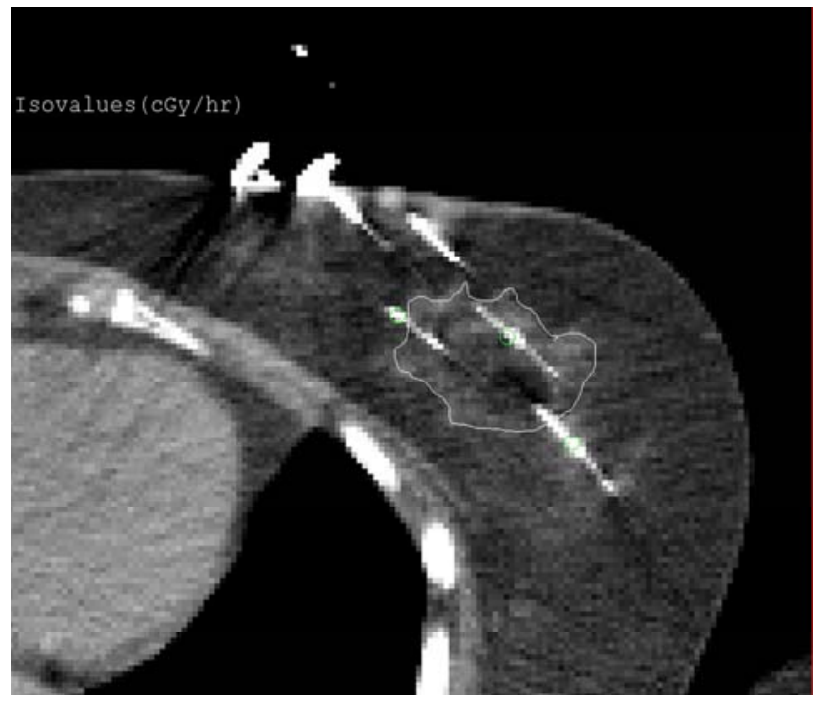

Figure 1. freehand tumor bed implantation was performed using afterloading catheters with the clinical target volume (CTV) consisting of the tumor bed/seroma cavity plus a minimum $1.0 \mathrm{~cm}$ margin

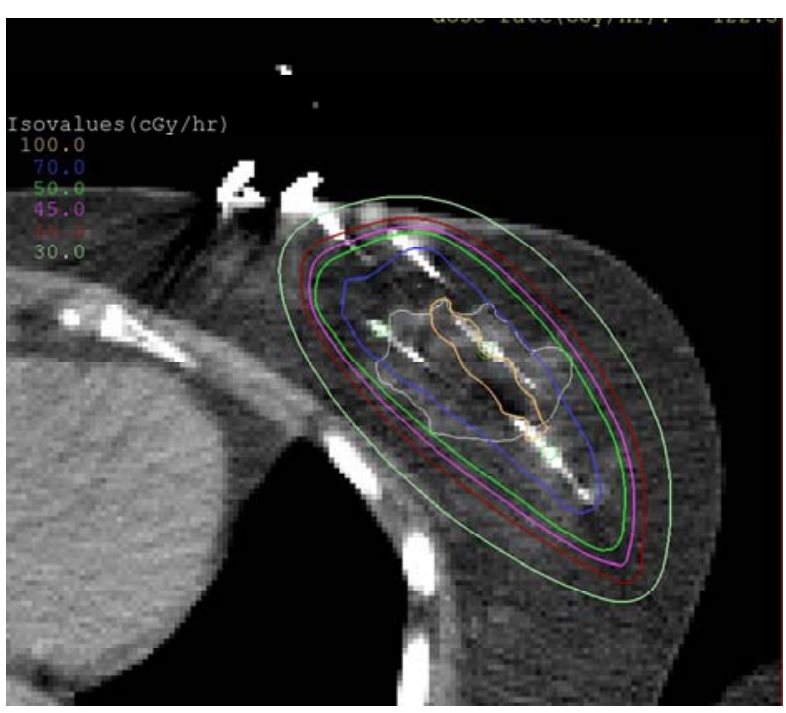

Figure 2. ${ }^{192}$ Iridium (mean activity $0.62 \mathrm{mg}$ Ra eq) was used in an afterloading inpatient procedure to achieve a target total dose of 4500-5000cGy, (range 4500-5530cGy) 


\section{Results}

Following treatment for their recurrent disease, twenty-one of 22 patients (95\%) were free of disease with a median follow-up of 67.5 months (range 17 - 115 months). Each of the patients had developed their IBTR approximately $1.0 \mathrm{~cm}$ from the skin surface and the implant treatment volume encompassed a significant portion of the previously treated skin. Two patients developed wound breakdown following repeat lumpectomy and implant, which subsequently healed by primary intention. The technique was reviewed and it was determined that the apparent skin dose may have been under represented by the treatment planning computer, therefore the procedure was modified shortly afterward to reduce skin dose and no additional patients developed grade 3 complications. No correlation between acute toxicity and final cosmetic outcome was identified.

One patient developed a second IBTR 23 months after her implant. She was treated for her initial disease in 1990 by lumpectomy and post-operative irradiation (5040cGy) to the entire breast with an additional tumor bed dose of 1440 cGy. The second IBTR occurred in the center of the high dose volume of the upper outer quadrant of the breast which received 5500cGy (V110) during her interstitial implant. Each time the margins of resection were negative and no extensive intraductal component was seen. Both the initial and first recurrent histology were DCIS. However, only the recurrence demonstrated comedonecrosis. She was subsequently successfully treated with salvage simple mastectomy and continues to be free of disease 7 years post second recurrence.

Two patients died from extensive metastatic disease at 17 and 24 months following their implant. One developed metastatic disease prior to brachytherapy, but was assessed as free of disease for two years following high dose chemotherapy and autologous bone marrow transplant prior to implant (the standard of care at that time). A second patient developed brain metastasis (not biopsied but likely breast cancer) 24 months after the brachytherapy implant.

A third patient succumbed to respiratory failure twelve months post implant secondary to complications arising from long-standing chronic pulmonary obstructive disease. A CT scan performed immediately prior to her demise did not demonstrate a pulmonary abnormality that would suggest irradiation insult. All patients who have died were free of local failure at the time of their deaths. When the patients who died from early disease were removed from the analysis, the mean follow up time increased to 74 months compared to 67.5 months.

Cosmetically, at their last follow-up, thirteen patients were scored using the Harvard scale (Table II) as grade 1, six as grade 2, and three as grade 3. No patient has received a Grade 4 score. The two patients with the most significant side effects of skin breakdown were finally categorized as Grade 2 cosmesis when healing was complete. Telangiectasia was noted in 5 patients. Five patients developed worsening cosmesis following radiation which was better reflected in the Allegheny General modification of the Harvard score (Table III). Two developed final cosmesis of $A^{[1,2]}$ while three were scored as $\mathrm{A}^{[2,3]}$. All long-term cosmesis stabilized at 2-2.5 years post-implant. A verbal survey of the 22 patients in this study was taken 6 months and one year post implant, and all were pleased they had undergone a breast sparing procedure as compared to mastectomy, including the single patient who developed a second IBTR.

\section{Discussion}

The accepted standard of care for IBTR has historically been salvage mastectomy which has demonstrated excellent single digit local recurrence rates ${ }^{[3,4,16,2-23]}$. While the local control is excellent, the cosmetic results are not. Women who have had mastectomy report significant impact upon their quality of life ${ }^{[5,24]}$. Even women who undergo post-mastectomy reconstruction report a lesser quality of life ${ }^{[25]}$. Lumpectomy only following IBTR is a suboptimal therapy, as patients who select observation alone after second lumpectomy face recurrence risks of $19-35 \%$ or higher ${ }^{[26,27]}$.

In our original study ${ }^{[13]}$, we demonstrated efficacy and safety in a non-randomized consecutive patient trial at a mean follow up of 35 months. Although other authors have confirmed our findings using differing dose regimens ${ }^{[14,15]}$, our LDR 
trial represents the largest cohort to date treated with uniform dose and prescription parameters. Our mature data at 67.5 months mean follow up continue to support the use of LDR brachytherapy as an acceptable alternative standard of care in lieu of mastectomy. Since our original report ${ }^{[13]}$, the cosmetic effects in all patients have remained stable. We note that cosmetic stability occurs at approximately 2-2.5 years from the brachytherapy. We also noted a severe limitation to the Harvard scoring system ${ }^{[18]}$ (Table I) in retreatment patients and introduced the Allegheny General Modification of the Harvard criteria in a previous publication (Table II) ${ }^{[19]}$. This scoring system is distinguished from the Harvard criteria by including an initial cosmetic score prior to re-irradiation and a final score after the second conservation procedure. This allows the cosmetic score to more accurately reflect the long term cosmetic effects of retreatment taking into account the initial cosmetic effect following the second surgical procedure. Post-operative de-novo cosmesis scores in our institution are nearly $100 \%$ with a cosmetic score of 1 following initial lumpectomy. However; re-treatment cosmesis scores prior to irradiation in this cohort were fifteen of grade 1 , six grade 2, and zero grade 3 . There were no cosmetic scores of 4 either prior to re-irradiation or following. Since three patients devolved from a cosmetic score of two to a score of three; and a score of three represents only fair cosmesis, the Allegheny Modification of the Harvard scale is critical in the understanding of the final cosmetic result and provides guidance in the selection of patients for retreatment. We suggest that patients who present for repeat conservation should preferably have a projected post repeat surgical score of 2 or lower to achieve satisfactory final cosmesis.

Despite the failure of conservation therapy in one patient and the increased cosmetic grade in five, all patients were pleased to have chosen breast conservation over mastectomy. We recommend thorough discussion with all patients prior to repeat conservative therapy, but patients who present with score 2 or higher should be cautioned about the possibility of worsening cosmesis and perhaps shown photos of prior patients who have shown devolution of cosmesis in order that the new patients have a clear an understanding as possible of the likely final outcome. In our previous publication we described a modification of technique and an improved selection process which aided in prediction of long-term cosmetic outcomes. ${ }^{13}$ Patients selected should have adequate breast tissue to accommodate the implant following repeat lumpectomy and the skin should be spared by placing the seeds at least $1.0 \mathrm{~cm}$ deep to the skin surface.

The issue of IBTR presents a challenging problem for the clinician and the patient alike. Historically many options have been offered for management of patients who develop localized breast recurrence. We continue to advocate for LDR brachytherapy as an acceptable option in lieu of salvage mastectomy in patients who desire breast preservation. However; emerging brachytherapeutic techniques such as balloon catheters may also represent promising alternatives ${ }^{[28,29]}$. While LDR brachytherapy provides for the best homogeneity among these option, the risks of a hospital acquired infection over the 4 - 5 day inpatient stay as well as the costs make newer options such as outpatient high dose rate brachytherapy or 3D-CRT more attractive. We recommend specific selection criteria for all patients for who repeat lumpectomy and retreatment irradiation is considered. First, the performance status of the patient must be evaluated, and the risks and benefits considered relative to the expected long term survival of the patient estimated in terms of both projected life expectancy respective and irrespective of the malignancy. Secondly, patients should have adequate residual tissue volume to support the implant and demonstrate projected acceptable post-operative cosmesis to accommodate a repeat surgical defect. Thirdly, we recommend retreatment only in patients who present with favorable tumor characteristics (tumor size $2.0 \mathrm{~cm}$. or less, negative final margins of resection, no clinically apparent lymphadenopathy, and no evidence of metastatic disease). Finally, all patients should understand clearly that mastectomy is the accepted standard of care.

\section{Conclusion}

Interstitial brachytherapy continues to prove itself at extended follow-up as a safe and effective modality for treatment of locally recurrent carcinoma of the breast in carefully selected subsets of patients. It offers equivalent local control to mastectomy. The procedure is well tolerated in the hands of an experienced brachytherapist and long term cosmesis remains stable after approximately 2-2.5 years. The Allegheny General Modification of the Harvard cosmesis scale gives a more accurate cosmetic reference and should be used in retreatment patients to more accurately report cosmetic 
outcomes. More importantly, patients remain satisfied with their outcomes especially as compared to the idea of mastectomy. The results of RTOG 1014 are eagerly awaited.

\section{Conflict of interest}

The author declares that there is no conflict of interest.

\section{References}

[1] Veronesi U, Cascinelli N, Mariani L, et al. Twenty-year follow-up of a randomized study comparing breast-conserving surgery with radical mastectomy for early breast cancer. N Engl J Med 2002;347:1227-32. PMid:12393819 http://dx.doi.org/10.1056/NEJMoa020989

[2] Fisher B, Anderson S, Bryant J, et al. Twenty-year follow-up of a randomized trial comparing total mastectomy, lumpectomy, and lumpectomy plus radiation for the treatment of invasive breast cancer. N Engl J Med 2002;347:1233-41. PMid:12393820 http://dx.doi.org/10.1056/NEJMoa022152

[3] Fowble BL, Solin LJ, Schultz DJ, et al. Ten year results of conservative surgery and irradiation for stage I and II breast cancer. Int J Radiat Oncol Biol Phys 1991;21:269-77. http://dx.doi.org/10.1016/0360-3016(91)90771-U

[4] Abner AL, Recht A, Eberlein T, et al. Prognosis following salvage mastectomy for recurrence in the breast after conservative surgery and radiation therapy for early-stage breast cancer. J Clin Oncol 1993;11:44-48. PMid:8418240

[5] Ganz P et al. Breast Conservation versus mastectomy; is there a difference in Psychological adjustment or quality of life in the year after surgery? Cancer 1992; 69:1729-38. http://dx.doi.org/10.1002/1097-0142(19920401)69:7<1729::AID-CNCR2820690714>3.0.CO;2-D

[6] Benitez PR, Keisch ME, Vicini F, et al. Five-year results: the initial clinical trial of MammoSite balloon brachytherapy for partial breast irradiation in early stage breast cancer. Am J Surg 2007;194:456-62. PMid:17826055 http://dx.doi.org/10.1016/j.amjsurg.2007.06.010

[7] Strnad V, Ott OJ, Hildebrandt G, et al. Partial breast irradiation using multicatheter interstitial brachytherapy for early breast cancer: Results of the German-Austrian multicenter Phase II trial. Brachytherapy 2009;8:107. http://dx.doi.org/10.1016/j.brachy.2009.03.011

[8] King TA, Bolton JS, Kuske RR, et al. Long-term results of wide-field brachytherapy as the sole method of radiation therapy after segmental mastectomy for Tis, 1, 2 breast cancer. Am J Surg 2000;180:299-304. http://dx.doi.org/10.1016/S0002-9610(00)00454-2

[9] Polgar C, Fodor J, Major T, et al. Breast-conserving treatment with partial or whole breast irradiation for low-risk invasive breast carcinoma - 5-year results of a randomized trial. Int J Radiat Oncol Biol Phys 2007;69:694-702. PMid:17531400

[10] Arthur DW, Winter K, Kuske RR, et al. A phase II trial of brachytherapy alone after lumpectomy for select breast cancer: tumor control and survival outcomes of RTOG 95-17. Int J Radiat Oncol Biol Phys 2008;72:467-73. PMid:18294778 http://dx.doi.org/10.1016/j.ijrobp.2007.12.056

[11] Vicini FA, Antonucci V, Wallace M, et al. Long-term efficacy and patterns of failure after accelerated partial breast irradiation: a molecular assay-based clonality evaluation. Int J Radiat Oncol Biol Phys 2007;68:341-46. PMid:17306933 http://dx.doi.org/10.1016/j.ijrobp.2006.12.007

[12] Polgar C, Major T, Fodor J, et al. Accelerated partial breast irradiation using high-dose-rate interstitial brachytherapy: 12-year update of a prospective clinical study. Radiother Oncol 2010; 94:274-79. PMid:20181401 http://dx.doi.org/10.1016/j.radonc.2010.01.019

[13] Trombetta M, Julian T, Bhandari T, et al. Breast conservation surgery and interstitial brachytherapy in the management of locally recurrent carcinoma of the breast: The Allegheny General Hospital experience. Brachytherapy 2008;7(1):29-36, January-March. PMid:18201940 http://dx.doi.org/10.1016/j.brachy.2007.12.001

[14] Chadha M, Feldman S, Boolbol S, et al. The feasibility of a second lumpectomy and beast brachytherapy for localized cancer in a breast previously treated with lumpectomy and radiation therapy for breast cancer. Brachytherapy. 2008 Jan-Mar; 7:22-28. PMid:18299110 http://dx.doi.org/10.1016/j.brachy.2007.10.006

[15] Hannoun-Levi JM, Houvenaeghel G, Ellis S, et al. Partial breast irradiation as second conservative treatment for local breast cancer recurrence. Int J Radiat Oncol Biol Phys. 2004;60:1385-92. PMid:15590169 http://dx.doi.org/10.1016/j.ijrobp.2004.05.035

[16] RTOG protocol 1014: A phase II study of repeat breast preserving surgery and 3D-conformal partial breast re-irradiation (PBRI) for local recurrence of breast carcinoma; http://www.rtog.org/ClinicalTrials/ProtocolTable/StudyDetails.aspx?study=1014

[17] http://evs.nci.nih.gov/ftp1/CTCAE/About.html 
[18] NSABP Protocol B-39/ RTOG Protocol 0413. A Randomized Phase III Study of Conventional Whole Breast Irradiation (WBI) Versus Partial Breast Irradiation (PBI) for Women with Stage 0, I, or II Breast Cancer; Form COS

[19] Trombetta M, Julian TB, Kim Y, et al. The Allegheny General Modification of the Harvard Breast Cosmesis Scale for the Retreated Breast. Oncology Vol.23 October 2009.

[20] Salvadori, B, Marubini E et al. Reoperation for Locally Recurrent Breast cancer in Patients Previously treated with conservative surgery. Br Journ Surg 1999,86,84-87. PMid:10027366 http://dx.doi.org/10.1046/j.1365-2168.1999.00961.x

[21] Huang E, Bucholz T, et al. Classifying local disease recurrences after breast conservation therapy based on location and histology. Cancer 2002; Vol. 95, No.10:2059-67. PMid:12412158 http://dx.doi.org/10.1002/cncr.10952

[22] Doyle T, Schultz D, et al. Long-term results of local recurrence after breast conservation treatment for invasive breast cancer. Int.J.Radiation Oncology Biol. Phys., 2001 Vol.51,No.1:74-80 http://dx.doi.org/10.1016/S0360-3016(01)01625-X

[23] Caucom C, Tsangaris T, et al. Results of salvage mastectomy for local recurrence after breast-conserving surgery without radiation therapy. Cancer 1993; Vol.57, No.5:1174-79.

[24] Maunsell E, et al. Psychological Distress after Initial Treatment for Breast Cancer: A Comparison of Partial and Total Mastectomy. J Clin Epidemiol 42:765-71,1989. http://dx.doi.org/10.1016/0895-4356(89)90074-7

[25] Rowland et al. Role of Breast Reconstruction in Physical and Emotional Outcomes among Breast Cancer Survivors. JNCI 2000; 92:1422-29. PMid:10974078 http://dx.doi.org/10.1093/jnci/92.17.1422

[26] Kurtz JM, et al. Results of salvage surgery for mammary recurrence following breast conserving therapy. Ann Surg 1988; 207:347-51 PMid:3345121 http://dx.doi.org/10.1097/00000658-198803000-00021

[27] Komoike Y, Motomura K, et al. Repeat lumpectomy for patients with ipsilateral breast tumor recurrence after breast conserving surgery. Oncology 2003; 64:1-6 PMid:12457024 http://dx.doi.org/10.1159/000066512

[28] Trombetta M, Julian TB, Miften M, McWilliams W, Kim Y, Parda D: The use of the MammoSite ® balloon applicator in re-irradiation of the breast. Brachytherapy 7(2008)316-19. PMid:18786865 http://dx.doi.org/10.1016/j.brachy.2008.06.001

[29] Trombetta M, Julian TB, Werts ED, Colonias A, Betler J, Kotinsley K, Kim Y, Parda D: Comparison of conservative management techniques in the retreatment of ipsilateral breast tumor recurrence. Brachytherapy;10(1):74-80, January-February 2011. PMid:20685174 http://dx.doi.org/10.1016/j.brachy.2010.01.005 